\title{
Hemangioma of the Maxillary Sinus Presenting as a Mass: CT and MR Features
}

\author{
Won Sang Jung ${ }^{1}$; Chang Young Yoo ${ }^{2}$; Yong-Jin Park ${ }^{3}$; Yon Kwon Ihn ${ }^{1, *}$ \\ ${ }^{1}$ Department of Radiology, St. Vincent's Hospital, The Catholic University of Korea, Suwon, Korea \\ ${ }^{2}$ Department of Pathology, St. Vincent's Hospital, The Catholic University of Korea, Suwon, Korea \\ 3 Department of Otorhinolaryngology, St. Vincent's Hospital, The Catholic University of Korea, Suwon, Korea \\ *Corresponding author:Yon Kwon Ihn, Department of Radiology, St. Vincent's Hospital, The Catholic University of Korea, Suwon, Korea. Tel: +82-312497491, E-mail:ihn@catholic.ac.kr
}

Received: July 18, 2012; Revised: January 18, 2013; Accepted: March 5, 2013

\begin{abstract}
Hemangiomas of the sinonasal tract are rare, and because these lesions lack the typical signs or symptoms, they can be confused with other malignant conditions. We report a case of cavernous hemangioma of the maxillary sinus in a 68-year-old man that was completely resected by endoscopic sinus surgery. Although computed tomography (CT) and magnetic resonance imaging (MRI) showed several enhancing areas within the tumor, the substantial bone erosion and remodeling made it difficult to differentiate this cavernous hemangioma from other expansile maxillary sinus lesions. We present the CT and MR findings of this lesion and discuss the differential diagnoses and potential therapeutic approaches.
\end{abstract}

Keywords: Hemangioma; Maxillary Sinus; Neoplasms; Vascular Tissue; Expansile Bone Lesions

\section{Introduction}

Although hemangiomas are relatively common in the head and neck, they occur infrequently in the sinonasal cavity (1-4). It is difficult to differentiate hemangiomas from more common expansile maxillary sinus lesions on the basis of the patient's symptoms and imaging findings. We report a case of maxillary sinus hemangioma and we discuss the imaging features, which may suggest the diagnosis.

\section{Case Presentation}

A 68-year-old male presented with longstanding, left sided nasal obstruction and serosanguinous nasal discharge. He had been experiencing nasal obstruction and intermittent epistaxis for one year already. His past medical history was otherwise unremarkable. The patient complained of the recent onset of left cheek fullness. On anterior rhinoscopy, a bulge was detected, arising from the left nasal wall and impinging on the septum. The computed tomography (CT) examination was performed on a spiral scanner (Light Speed VCT, GE Medical Systems, Milwaukee, WI, USA). The CT scan obtained following intravenous contrast administration revealed a large, inhomogeneously enhancing mass in the left maxillary sinus and nasal cavity (Figure $1 \mathrm{~A}$ and B). The nasal turbinates and the medial wall of the maxillary sinus had been eroded by the mass. There was also a bony remodeling in the anterior wall of the maxillary sinus. For further evaluation, magnetic resonance imaging(MRI) was performed on a 1.5 T scanner (Gyroscan NT; Philips, Eind- hoven, Netherlands) to produce pre- and post-contrast T1weighted spin-echo images and T2-weighted fast spin-echo images, with or without fat saturation. The MR images were obtained in at least two planes with $5 \mathrm{~mm}$ section thickness and 0.5 - $1.5 \mathrm{~mm}$ intersection gap. The MRI showed a heterogeneous high-signal intensity mass on the T2-weighted images (Figure 2). When enhanced with gadolinium (our institute's routine MRI protocol of our institute includes fat suppression for axial enhanced T1-weighted image), it seemed to arise from the antrum of the maxillary sinus and extended into the left nasal cavity, through the ostiomeatal complex (Figure 3). Because of the suspicion of sinonasal cancer, a biopsy was taken to obtain tissue from the nasal cavity and maxillary sinus. However, the biopsy revealed only stromal sclerosis and chronic necrotizing inflammation. The tumor was completely excised by performing endoscopic sinus surgery with the patient under general anesthesia. No significant hemorrhage occurred during the surgery, and the surgical findings were well correlated with the CT imaging. However, the uncinate processes as well as the medial wall of the maxillary sinus were found to have been destroyed by the mass. The microscopic examination showed dilated sinusoidal vascular channels in the stroma lining with an interconnecting fibrous wall covered with flat endothelium. The lumina of these spaces were filled with blood. The histologic features were most compatible with cavernous hemangioma (Figure 4). On follow up after 1 year, the patient was asymptomatic, without any sign of recurrence.

Copyright (C) 2015, Tehran University of Medical Sciences and Iranian Society of Radiology. This is an open-access article distributed under the terms of the Creative Commons Attribution-NonCommercial 4.0 International License (http://creativecommons.org/licenses/by-nc/4.0/) which permits copy and redistribute the material just in noncommercial usages, provided the original work is properly cited. 

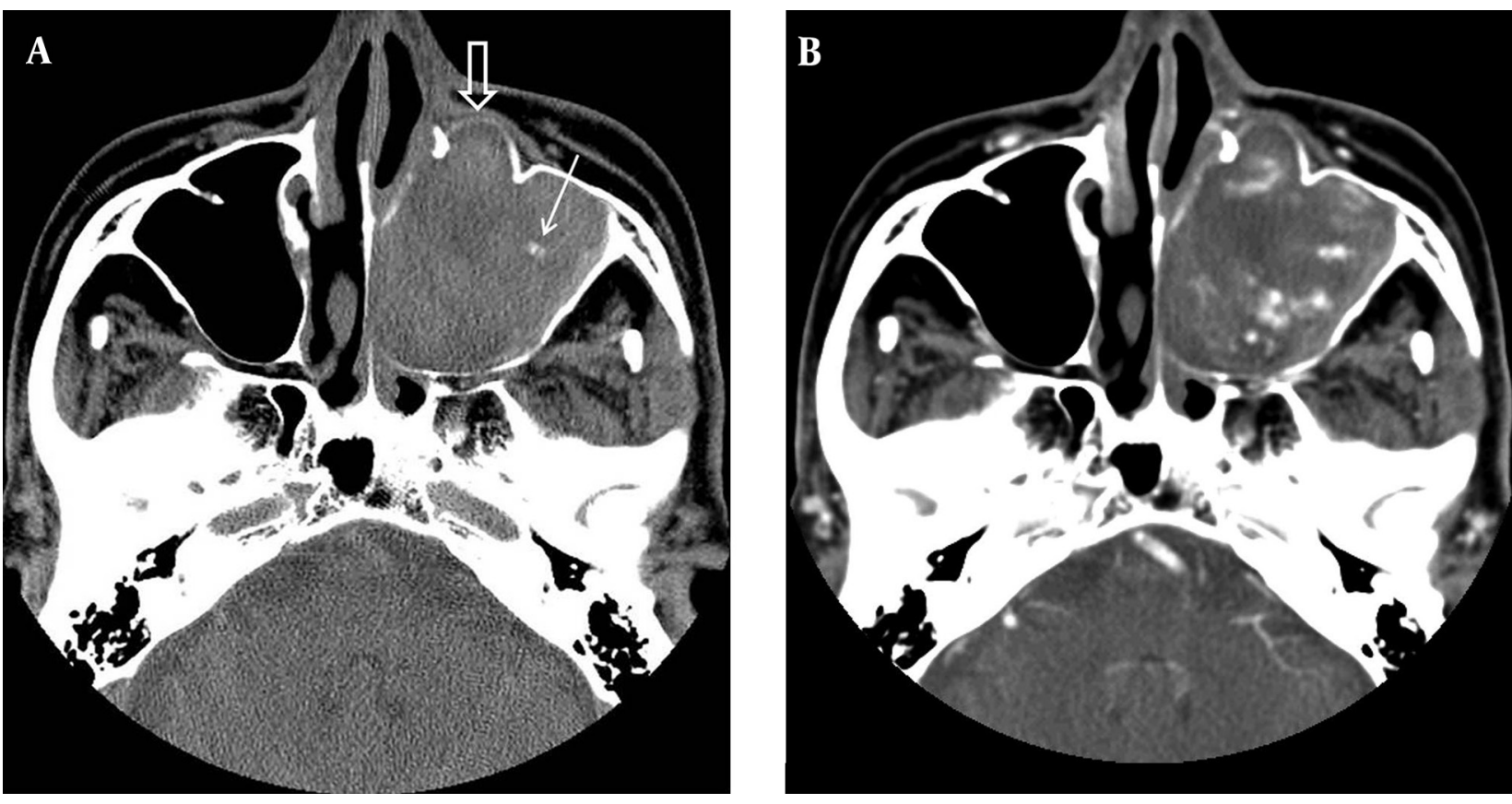

Figure 1. CT images of A 68-year-old man with maxillary sinus hemangioma. A, Nonenhanced axial CT scan shows a large, soft tissue mass in the nasal cavity and maxillary sinus, on the left side. The medial wall of the maxillary sinus has been eroded by the mass. Note the focal bone remodeling of the anterior wall of the left maxillary sinus (open arrow). There is a small calcification in the maxillary sinus lesion (arrow). B, Enhanced axial CT of the lesion shows an inhomogeneously enhancing soft tissue mass.

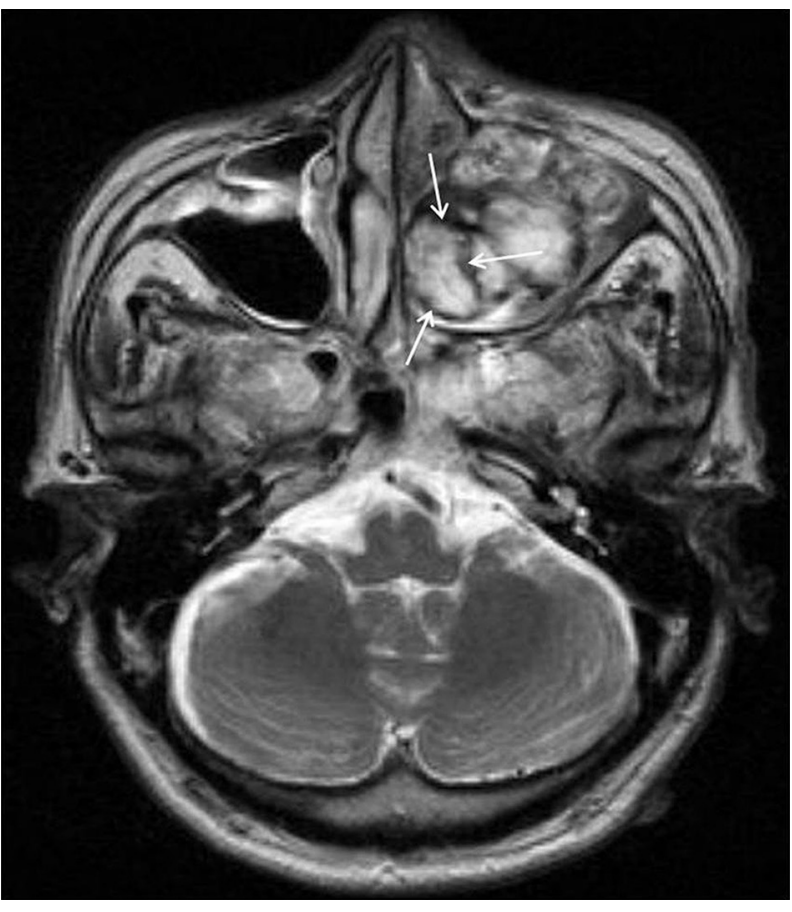

Figure 2. T2-weighted magnetic resonance imaging of the mass. Axial T2weighted MR image shows a high signal intensity mass in the left maxillary sinus, which is surrounded by a low-signal intensity rim (arrows)

\section{Discussion}

Hemangiomas of the head and neck are benign vascular lesions, and although they are common in these locations, they occur infrequently in the paranasal sinuses. Hemangiomas are divided into capillary and cavernous types, depending on the dominant vessel size seen on microscopic examination. Capillary hemangioma is the more common type and is composed of capillary-sized vessels lined with flattened epithelium. Cavernous hemangiomas are composed of large, endothelium-lined vascular spaces (5). The common symptoms of the sinonasal hemangiomas include nasal obstruction, epistaxis, and, occasionally, a visible nasal mass (6). Although there have been multiple clinical studies of nasal hemangioma (2), only less than 20 cases of maxillary hemangioma have been reported $(1,3)$.

The radiographic appearance of hemangiomas has been reported in the literature. Previously reported CT features of maxillary sinus hemangioma can be summarized as depicting a highly vascularized, soft-tissue mass. Although several enhancing portions have been noted on CT scans, even larger areas did not enhance because of necrosis and hemorrhage within the tumor $(3,4)$. Furthermore, hemangiomas have been reported to cause changes in adjacent bone. According to Dillon et al. capillary hemangiomas of the nasal vault had benign appearing bone changes, consisting of remodeling or expansion (7). 

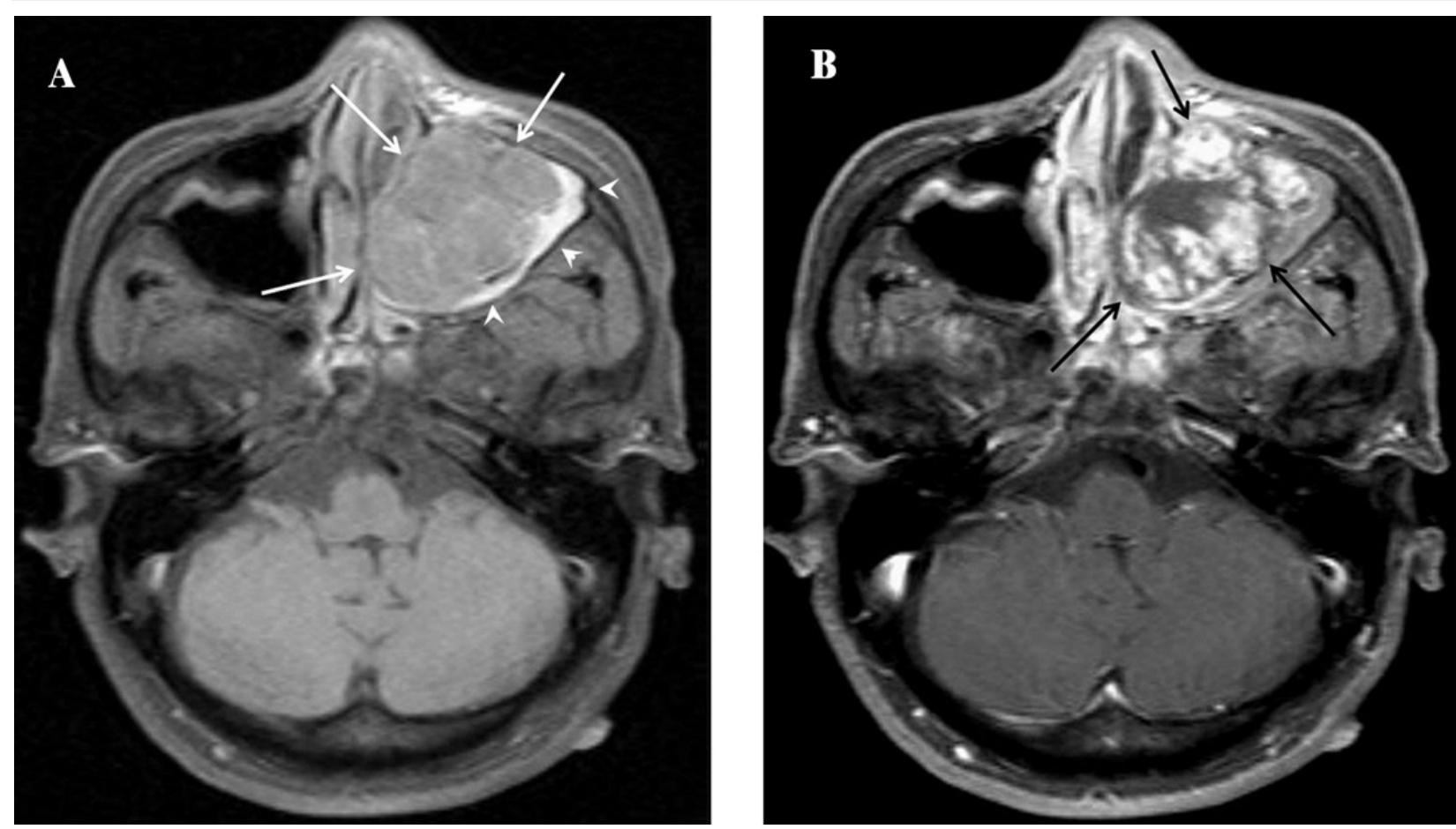

Figure 3. A, Axial fat-supressed, T1-weighted image shows intermediate signal intensity lobulating mass lesion (white arrows) in the left maxillary sinus. Obstructed sinonasal secretions in the left maxillary sinus have high signal intensity (arrowheads). B, Enhanced T1-weighted image shows heterogeneous intense enhancement (black arrows)."
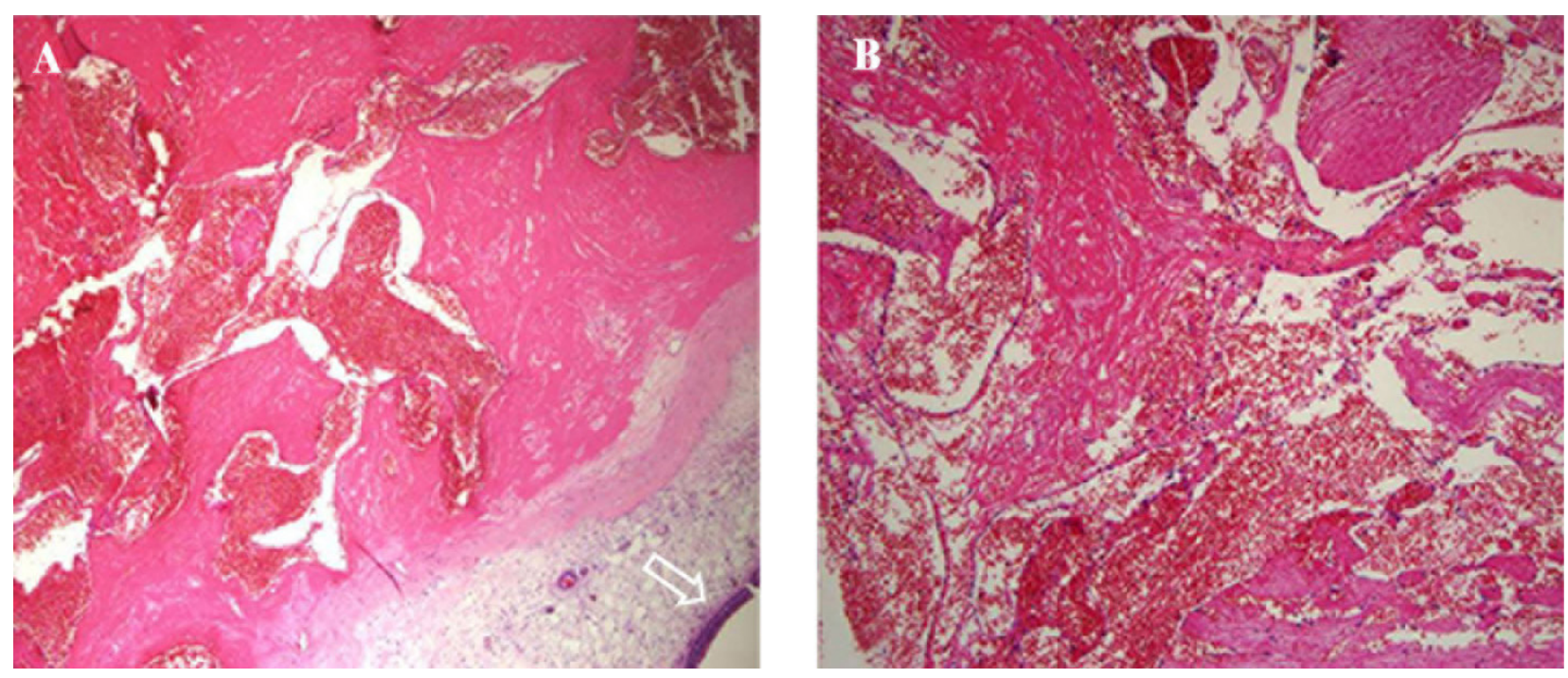

Figure 4. Histopathological view of the resected tumor. Histopathology reveals that a cavernous hemangioma is characterized by aggregation of large, thin-walled, dilated blood vessels. These vessels are separated by scant fibrous stroma. Intact surface epithelium of the respiratory mucosa is seen in the periphery (arrow). A: Hematoxylin \& Eosin staining; original magnification $\times 40$. B: Hematoxylin \& Eosin staining; original magnification $\times 100$.

Kim et al. reported two cases of cavernous hemangioma that caused erosion of the wall of the maxillary sinus, nasal turbinate, and orbit (8). According to Weiss et al. amorphous or curvilinear calcification is a non-specific finding, whereas phlebolith formation is a more specific finding of cavernous hemangioma (5). In our case, CT also showed a soft tissue mass, which caused expansile bone remodeling of the maxillary sinus, with a small calcification within the lesion, similar to previously reported CT findings. We, therefore, assume that this CT finding offers a diagnostic clue regarding the presence of a hemangioma. According to Itoh et al. (9), MR images 
of cavernous hemangioma reflect the signal of unclotted blood, and they have prolonged $\mathrm{T} 1$ and $\mathrm{T} 2$ relaxation times, which result in low signal intensity on T1-weighted images and high signal intensity on T2-weighted images. However, these lesions do not show any of the signal voids associated with the hypervascularity, often seen in other types of vascular malformation. Dillon et al. reported that, on T2-weighted sequences, capillary hemangioma of the nasal vault shows a hypointense rim surrounding a mass of mixed signal intensities. Pathology examination revealed that these signals were apparently correlated with areas of clotted blood (7). Our patient's MR scan also showed a peripheral, hypointense rim, surrounding a central mass, on the T2-weighted MR sequence. Although an exact MR-pathology correlation could not be obtained, because the lesion was removed in pieces, we believe that the heterogeneous signal intensity seen on the MR images reflects the various components, such as hemorrhage, fibrosis, and vascular proliferation.

Organized hematoma $(\mathrm{OH})$ is the most difficult lesion to differentiate, both clinically and radiologically, from sinonasal cavernous hemangioma. Although Yagisawa et al. suggested that hemangioma and sinonasal $\mathrm{OH}$ are the same pathologic entity, the fact that the vascular lumina of cavernous hemangioma are usually larger than those of $\mathrm{OH}$ on histologic examination still raises the question of the probability of the different nature of the two lesions (10). In addition to $\mathrm{OH}$, mucocele, fungus ball, inflammatory polyp, cholesterol granuloma, inverted papilloma, and carcinoma can present as an expansile mass (11). Contrast material administration is useful because mucocele, fungus ball, polyp, and cholesterol granuloma are not usually enhanced. Carcinoma has a pattern of nodular enhancement (12).

The surgical resection is the main treatment for maxillary sinus hemangioma (4). According to tumor extent , different surgical resection methods can be used for maxillary sinus hemangioma, such as lateral rhinotomy, the Caldwell-Luc operation or endoscopic sinus surgery (4). Preoperative transarterial embolization can decrease the tumor size and reduce the risk of hemorrhage during surgery (6).

In conclusion, sinonasal cavernous hemangioma may be mistaken for a locally aggressive neoplasm. The CT and MR images have demonstrated an expansile, well-enhanced heterogeneous mass, with bone remodeling and erosive change. Internal calcification may also be visible. Although it is a very unusual tumor, based on the imaging findings, cavernous sinus hemangioma should be considered in the differential diagnosis of a paranasal sinus tumor.

\section{References}

1. Raboso E, Rosell A, Plaza G, Martinez-Vidal A. Haemangioma of the maxillary sinus. J Laryngol Otol. 1997;111(7):638-40.

2. Iwata N, Hattori K, Nakagawa T, Tsujimura T. Hemangioma of the nasal cavity: a clinicopathologic study. Auris Nasus Larynx. 2002;29(4):335-9.

3. Jammal H, Barakat F, Hadi U. Maxillary sinus cavernous hemangioma: a rare entity. Acta Otolaryngol. 2004;124(3):331-3.

4. Kanazawa T, Inoue R, Ohta Y, Watanabe Y, Iino Y. Maxillary haemangioma successfully resected by endoscopic approach. J Laryngol Otol. 2009;123(7):793-5.

5. Weiss SW, Enzinger FM, Goldblum JR. Enzinger and weiss's soft tissue tumors. 5 edLouis: Mosby; 2008.

6. Song CE, Cho JH, Kim SY, Kim SW, Kim BG, Kang JM. Endoscopic resection of haemangiomas in the sinonasal cavity. J Laryngol Otol. 2009;123(8):868-72.

7. Dillon WP, Som PM, Rosenau W. Hemangioma of the nasal vault: MR and CT features. Radiology. 1991;180(3):761-5.

8. Kim HJ, Kim JH, Kim JH, Hwang EG. Bone erosion caused by sinonasal cavernous hemangioma: CT findings in two patients. AJNR Am J Neuroradiol.1995;16(5):1176-8.

9. Itoh K, Nishimura K, Togashi K, Fujisawa I, Nakano Y, Itoh H, et al. MR imaging of cavernous hemangioma of the face and neck. $J$ Comput Assist Tomogr. 1986;10(5):831-5.

10. Yagisawa M, Ishitoya J, Tsukuda M. Hematoma-like mass of the maxillary sinus. Acta Otolaryngol. 2006;126(3):277-81.

11. Lee HK, Smoker WR, Lee BJ, Kim SJ, Cho KJ. Organized hematoma of the maxillary sinus: CT findings. AJR Am J Roentgenol. 2007;188(4):W370-3.

12. Som PM, Branwein MS, Kassel EE, Genden EM. Tumors and tumor like conditions of the sinonasal cavities. 5 edLouis: Mosby; 2011. 